\title{
Shunt atriocava. A propósito de dos casos
}

\author{
Sebastián Soto $\mathbf{G}^{1}$, Roberto Oettinger $\mathbf{W}^{1-2}$. \\ Atriocaval shunt. Report of two cases
}

The mortality of grade V and VI liver trauma fluctuates between $30 \%$ and $70 \%$. The atriocaval shunt, described by Shrock et al, in 1968, is a therapeutic option that, after being installed, allows to repair the suprahepatic veins and retrohepatic cava in a bloodless surgical field. Its use requires an experienced and skilled surgeon to obtain survival rates similar to those obtained with other methods. We report two male patients of 17 and 18 years old treated successfully with this technique after suffering a blunt and a penetrating liver trauma by a shotgun, respectively (Rev Méd Chile 2005; 133: 327-30).

(Key Words: Arteriovenous shunt, surgical; Hepatic veins; Vena cava, superior)

Recibido el 24 de agosto, 2004. Aprobado el 18 de noviembre, 2004.

${ }^{1}$ Instituto de Cirugía, Facultad de Medicina, Universidad Austral de Chile.

${ }^{2}$ Servicio de Cirugía, Hospital Clínico Regional de Valdivia. Valdivia, Chile.

$\mathrm{E}^{\mathrm{n}}$ cirugía de urgencia existen situaciones que requieren un amplio manejo de opciones terapéuticas, como también de una rápida elección y realización. Entre estas situaciones se incluye al hígado, que a pesar de estar relativamente protegido dentro del abdomen, en trauma grado V y VI, se lesionan venas suprahepáticas o cava retrohepática, produciéndose una hemorragia exanguinante con mortalidad variable entre $30 \%$ y $70 \%{ }^{1}$, antes de recibir asistencia. Una de las opciones terapéuticas es la instalación de un shunt atriocava ${ }^{2-3}$.

A pesar de las dificultades técnicas que pudiese presentar, sigue siendo útil, permitiendo salvar vidas, como se ha reportado previamente en la literatura médica ${ }^{1-8}$, sin embargo, no está exento de mortalidad intraoperatoria $(50 \%-90 \%)^{3}$, princi-

Correspondencia a: Sebastián Soto G. Pérez Rosales 1035, Valdivia. Fax: (63) 221931. E-mail: seba_soto_g@yahoo.es palmente, por complicaciones del paciente con trauma severo y politransfundido, con la triada letal de coagulopatía, hipotermia y acidosis.

En manos entrenadas, ha demostrado tener resultado con sobrevida variable entre $15 \%$ y $25 \%$, similar a la obtenida con técnicas menos invasivas $^{1-8}$.

Consideramos de interés comunicar los casos de dos pacientes de sexo masculino con trauma hepático grado $\mathrm{V}$ tratados en forma exitosa con esta técnica.

\section{TÉCNICA QUIRÚRGICA}

Identificada la lesión hepática grado $\mathrm{V}$, se amplía la incisión a toracofrenolaparotomía derecha, pericardiotomía longitudinal y ampliación digital, identificación de la orejuela, gareta de material no reabsorbible, apertura de la misma e introducción 
hacia vena cava inferior de tubo orotraqueal 7,5, previa fenestración lateral, que permite flujo hacia la aurícula, se avanza quedando distal a las lesiones venosas a reparar, por sobre las renales y se insufla el manguito, canalizando el flujo de la cava inferior a través del tubo a la aurícula derecha. El extremo proximal del tubo queda por fuera de la orejuela y se clampea (Figura 1). Terminada la reparación se retira tubo y se cierra la gareta.

\section{CASO CLÍNICO 1}

Hombre de 17 años sin antecedentes mórbidos, que ingresó el 24/07/1992, a la Unidad de Emergencia del Hospital Clínico Regional de Valdivia, 20 minutos posterior a haber sufrido trauma toracoabdominal cerrado (aplastamiento entre muralla de

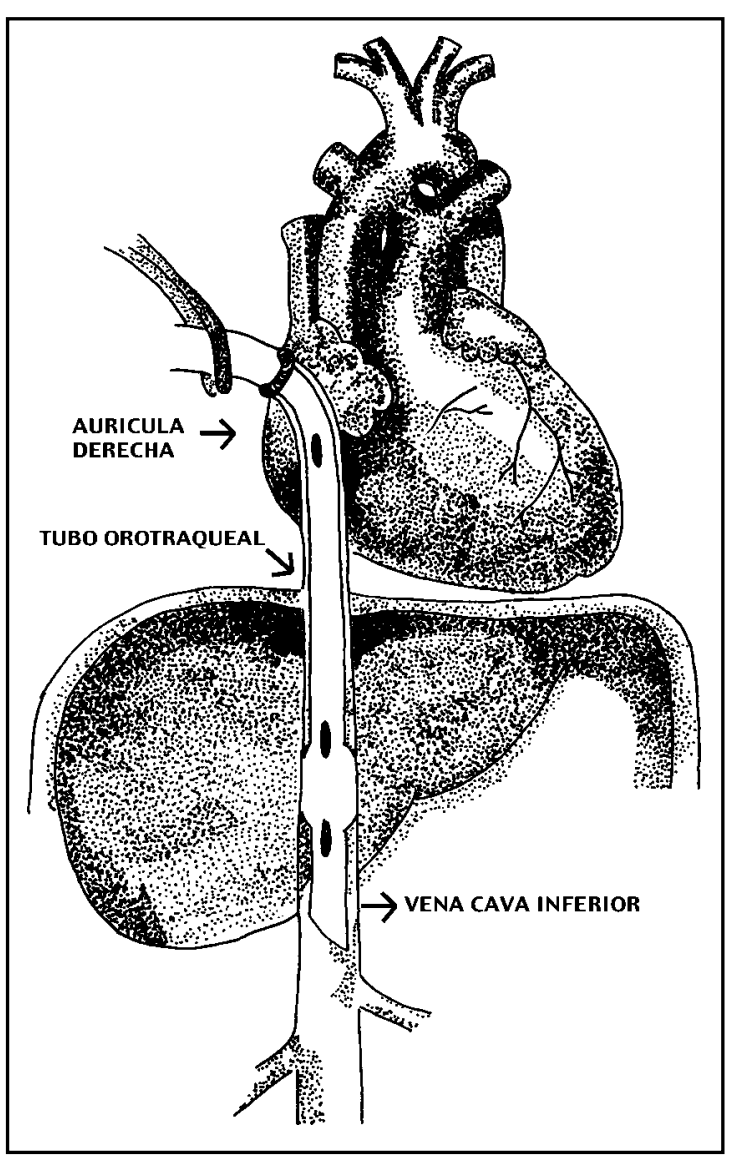

FIgURA 1. concreto y camión). Al examen, se encontró conciente, orientado temporoespacialmente, pálido, taquicárdico (pulso 112x' débil), hipotenso (PA 70/ $50 \mathrm{mmHg}$ ), respiración paradojal, destacando múltiples erosiones toracoabdominales, crepitaciones costales múltiples, aumento de volumen y deformidad del antebrazo izquierdo, abolición del murmullo pulmonar en base derecha, abdomen sensible y pérdida de la matidez hepática. Se efectuó ecografía abdominal que mostró líquido libre, sin identificar lesión hepática.

Una vez estabilizado, fue a pabellón, a las $2 \mathrm{~h}$ después del ingreso, con los diagnósticos de trauma toracoabdominal complicado, probable rotura de víscera hueca, fractura de antebrazo izquierdo, fractura esternal, tórax volante y hemoneumotórax derecho. Se instaló pleurostomía derecha, dando salida a aire y sangre. Se abordó vía laparotomía media supraumbilical, encontrando hemoperitoneo de 3 l, se identificó lesión hepática única, paralela a ligamento falciforme, que seccionaba al hígado en dos. No se logró control del sangrado con maniobra de Pringle, identificando origen de sangrado en vena cava supra y retrohepática.

Se decidió realizar shunt atriocava, ampliando incisión a toracofrenolaparotomía derecha y se instaló tubo orotraqueal 7,5 a través de aurícula derecha, cediendo el sangrado, identificando 2 lesiones en vena cava suprahepática de 1 y $2 \mathrm{~cm}$ y una lesión de $1 \mathrm{~cm}$ a nivel de cava retrohepática, que se suturan con prolene $4 / 0$, completando, posteriormente, hepatectomía izquierda. Durante la cirugía se transfundieron $10 \mathrm{U}$ de sangre total, 3 U de glóbulos rojos, $5 \mathrm{U}$ de crioprecipitados, $2 \mathrm{U}$ de plaquetas y $6 \mathrm{U}$ de plasma.

Ingresó a la Unidad de Cuidados Intensivos (UCI) con PA 100/60 mmHg, pulso $162 \mathrm{x}$ ', PVC 16 $\mathrm{cmH}_{2} \mathrm{O}$. Se conectó a ventilación mecánica que se mantuvo por $48 \mathrm{~h}$, cursando posteriormente con coagulación intravascular. Evolucionó favorablemente, siendo trasladado al décimo día postoperatorio (03/08/1992) al Servicio de Cirugía y posteriormente a mutualidad el 08/08/1992, desde donde fue dado de alta en buenas condiciones el 20/08/1992.

\section{CASO CLÍNICO 2}

Hombre de 18 años sin antecedentes mórbidos que ingresó el 17/12/2001, a la Unidad de Emer- 
gencia del Hospital Clínico Regional de Valdivia, 2 $\mathrm{h}$ después de sufrir una herida a bala en región epigástrica. Al examen se encontraba conciente, orientado temporoespacialmente, hemodinámicamente estable (PA 120/70 mmHg, pulso $70 \mathrm{x}$ ), destacando herida epigástrica con escaso sangrado y signos de irritación peritoneal a la palpación (Blumberg y resistencia muscular).

Cuarenta y cinco minutos después de su llegada, ingresó a pabellón, con el diagnóstico de herida por arma de fuego abdominal complicada. Se abordó, vía laparotomía media supraumbilical, encontrando hemoperitoneo de 2,5 l, identificando herida anfractuosa de la cúpula de lóbulo hepático izquierdo, que sangraba profusamente, cediendo parcialmente con maniobra de Pringle y compresión directa. Se sospechó lesión de vena cava retrohepática, y se decidió shunt atriocava, ampliando incisión a toracofrenolaparotomía derecha e instalación de tubo orotraqueal 7,5 a través de la aurícula derecha, controlando el sangrado. Dada la magnitud de la lesión hepática, se realizó hepatectomía izquierda, encontrando lesión transfixiante de cava inferior que se reparó con prolene 4/0 corrido. Se dejó packing de la zona hepática cruenta, drenajes pleurales derechos y cierre. Durante el acto quirúrgico se transfundieron 12 U de glóbulos rojos, $1.324 \mathrm{cc}$ de plasma y $10 \mathrm{l}$ de coloides y cristaloides.

Ingresó a UCI estable hemodinámicamente, PA 139/65 mmHg, pulso 123 x', FR 21 x'. Se conectó a ventilación mecánica, que se mantuvo por $24 \mathrm{~h}$, cursando con coagulación intravascular, que requirió transfusión de crioprecipitados, evolucionando favorablemente.

El 20/12/2001 reingresó a pabellón, realizando aseo peritoneal y retiro de packing, sin evidencias de sangrado. Al $4^{0}$ día postoperatorio (21/12/ 2001) se trasladó al Servicio de Cirugía siendo dado de alta en buenas condiciones el día 29/12/ 01.

$\mathrm{Al} \mathrm{30/07/2004,} \mathrm{ambos} \mathrm{pacientes} \mathrm{se} \mathrm{encuentran}$ realizando sus actividades laborales habituales, sin secuelas funcionales.

\section{DisCUSIÓN}

El trauma hepático mayor (grado V, VI) es una situación compleja y devastadora a la que, ocasionalmente, se ve enfrentado el cirujano en urgen- cia. Se traduce en una hemorragia exanguinante, con alta mortalidad durante el traslado o durante la cirugía, lo que no ha mejorado durante los últimos años a pesar de la introducción de nuevas tecnologías ${ }^{9}$. Según Buckman y cols ${ }^{5}$, son múltiples los factores que inciden en la alta mortalidad de estas lesiones, incluyendo factores anatómicos, inexperiencia del equipo quirúrgico y estrategias terapéuticas poco acertadas, adicionalmente, enfermos que en épocas pasadas fallecían en el lugar del accidente, hoy ingresan vivos a las unidades de emergencia dadas las mejoras en la atención prehospitalaria ${ }^{5-9}$.

Las lesiones vasculares de las venas suprahepáticas y vena cava retrohepática significan un especial desafío para el cirujano, dada la dificultad técnica para lograr buena exposición como control proximal y distal.

Enfrentado al dilema anterior, Shrock y cols, mediante la disección en cadáveres, observaron que la vena cava inferior, sobre las renales, sólo recibe como tributarias la vena suprarrenal derecha, las venas suprahepáticas y las venas diafragmáticas inferiores, en base a lo cual, diseñaron un sistema que permite mantener el retorno venoso a la aurícula, mientras se reparan las lesiones de venas suprahepáticas o cava retrohepática, siendo conocido como shunt atriocava ${ }^{3,9,10}$. El mismo grupo lo llevó a la práctica clínica en 1968, sin lograr sobrevida del enfermo, lo anterior incentivó a otros, logrando Bricker y cols en 1970 el primer resultado exitoso ${ }^{3,11}$. En los años siguientes, el uso de la técnica se amplió a lo largo de los centros de trauma del mundo pero sobre todo dentro de Estados Unidos, con resultados positivos en casos aislados, demostrando ser reproducible en manos entrenadas ${ }^{3}$. Dada su escasa utilización, existen sólo 2 series con un número mayor de casos, Rovito y cols, en 1987, reportaron nueve casos con sobrevida de $44 \%$ (4 casos) ${ }^{3,12}$, posteriormente, en 1988, Burch y cols publicaron la mayor serie de la literatura, tratando 31 enfermos con una sobreviva de $19 \%$ (6 casos) ${ }^{3}$, valor vigente en la actualidad en este tipo de lesiones.

La técnica original describe que, posterior al control del sangrado mediante compresión, se amplía la laparotomía media supraumbilical con esternotomía, logrando amplia exposición de corazón y grandes vasos, se mide en forma externa la longitud del shunt, se realiza gareta en la 
orejuela y se introduce tubo pleural u orotraqueal, previamente fenestrado, quedando clampeado el extremo proximal por fuera de la orejuela, al pasar hacia distal, el cirujano debe tener cuidado de que el manguito quede por sobre las renales, para permitir drenaje de éstas y así, asociado a una maniobra de Pringle, lograr un campo quirúrgico casi exangüe, permitiendo la reparación de las lesiones.

En los 2 casos presentados se modifica la técnica original, ampliando el abordaje de laparotomía media supraumbilical a toracofrenolaparotomía derecha, por preferencia y comodidad del cirujano con este tipo de abordaje.

\section{REFERENCIAS}

1. Kiein SR, Baumgartner FJ, Bongard FS. Contemporary management strategy for major inferior vena caval injuries. J Trauma 1994; 37: 35-41.

2. Parks RW, Chrysos E, Diamond T. Management of liver trauma. Br J Surg 1999; 86: 1121-35.

3. Burch JM, Feliciano DV, Mattox KL. The Atriocaval Shunt Facts and Fiction. Ann Surg 1988; 207: $555-68$

4. David Richardson J, Frankin GA, Lukan JK, CarriLo EH, Spain DA, MuLer FB et aL. Evolution in the Management of Hepatic Trauma: A 25-Year Perspective. Ann Surg 2000; 232: 324-30.

5. Buckman RF JR, Miraliakbari $R$, Badeluno MM. Juxtahepatic Venous Injuries: A Critical Review of Reported Management Strategies. J Trauma 2000; 48: 978-84.

6. Coughin PA, Stringer MD, Lodge JP, Polatrd SG, PRASAD KR, Toogood GJ. Management of blunt liver trauma in a tertiary referral centre. Br J Surg 2004; 91: 317-21.

7. Hansen CJ, Bernadas C, West MA, Ney AL, MuehlsTEDT S, Cohen M et aL. Abdominal vena caval injuries: Outcomes remain dismal. Surgery 2000; 128: 572-8.

8. Marr JD, Krige JE, Terblanche J. Analysis of 153 gunshot wounds of the liver. Br J Surg 2000; 87: 1030-4.
En la actualidad existen múltiples alternativas al shunt atriocava, tal como el clampeo directo por sobre y bajo la lesión, técnicas endovasculares, el uso de circulación extracopórea en centros que cuentan con ella, el uso de shunt intravasculares, tal como los utilizados en trauma vascular periférico, o incluso el manejo conservador mediante ligadura propuesto por Patcher y cols en 1986, sin embargo esto no se traduce en mejoras en la sobrevida1,2-17. En manos entrenadas esta técnica sigue siendo útil, permitiendo salvar vidas a escala similar que con medidas conservadoras, lo que se refleja en los resultados obtenidos en los 2 casos en que ha sido utilizado en nuestro grupo.

9. Angeles AP, Agarwal N, Lynd C JR. Repair of a Juxtahepatic Inferior Vena Cava Injury Using a Simple Endovascular Technique. J Trauma 2004; 56: 918-21.

10. Schrock T, Blaisdell FW, Mathewson C Jr. Management of blunt trauma to the liver and hepatic veins. Arch Surg 1968; 96: 698-704.

11. BRICKER DL, WuKASCH DC. Successful management of an injury to the suprarenal inferior vena cava. Surg Clin North Am 1970; 50: 999-1002.

12. Rovito PF. Atrial caval shunting in blunt hepatic vascular injury. Ann Surg 1987; 205: 318-21.

13. SHARP KW, LOCICERo RJ. Abdominal Packing For Surgically Uncontrollable Hemorrhage. Ann Surg 1992; 215: 467-74.

14. Baumgartner F, Scudamore C, Nair C, Karusseit O, Hemming A. Venovenous Bypass for Major Hepatic and Caval Trauma. J Trauma 1995; 39: 671-73.

15. Erzurum VZ, Shoup M, Borge M, Kalman PG, Rodríguez $\mathrm{H}$, Silver GM. Inferior vena cava endograft to control surgically inaccessible haemorrhage. J Vasc Surg 2003; 38: 1437-9.

16. Soto S, Oettinger W, Brousse J, Sánchez G. Cirugía de control de daños. Enfrentamiento actual del trauma. Cuad Cir 2003; 17: 95-102.

17. Patcher HL, Spencer FC, Hofstetter SR, Luang HC, CopPa GF. The management of juxtahepatic venous injuries without an atriocaval shunt: preliminary clinical observations. Surgery 1986; 99: 569-75. 